\title{
CRITICAL ANALYSES OF NATURAL LANGUAGE PROCESSING (NLP) IN UNDERSTANDING SEMANTICS
}

\author{
Farida Huseynova \\ Azerbaijan State Oil and Industry University, Azerbaijan
}

DOI: https://doi.org/10.31435/rsglobal_ws/30092019/6695

\section{ARTICLE INFO}

Received: 15 July 2019

Accepted: 14 September 2019

Published: 30 September 2019

\section{KEYWORDS}

understanding, semantic aspects, computer languages, natural language.

\begin{abstract}
Today, language understanding systems do quite many useful things with processing natural language, even they are able to process the data much faster than humans are. Nevertheless, they do not have the same logical understanding of natural language yet as humans have and the interpretation capabilities of a language understanding system depending on the semantic theory is not sufficient in all aspects. The research is centered on some of the important issues that arise using it in natural language processing.
\end{abstract}

Citation: Farida Huseynova. (2019) Critical Analyses of Natural Language Processing (NLP) in Understanding Semantics. World Science. 9(49), Vol.1. doi: 10.31435/rsglobal_ws/30092019/6695

Copyright: (C) 2019 Farida Huseynova. This is an open-access article distributed under the terms of the Creative Commons Attribution License (CC BY). The use, distribution or reproduction in other forums is permitted, provided the original author(s) or licensor are credited and that the original publication in this journal is cited, in accordance with accepted academic practice. No use, distribution or reproduction is permitted which does not comply with these terms.

"Humans have many remarkable capabilities. Among them there are two that stand out in importance. First, the capability to converse, communicate, reason and make rational decisions in an environment of imprecision, uncertainty, incompleteness of information and partiality of truth

Introduction. Human always had curiosity and the desire to understand and influence the environment and very long time he craved to invent a human like device and at last, he developed his intelligent human aid, which emulated a person following a series of logical instructions. Subsequently, high-level computer languages also had been developed for achieving different variety of tasks.

Today, computers do quite many useful things with processing natural language, even they are able to process the data much faster than humans are, but they do not have the same logical understanding of natural language yet as humans.

It is true that many AI projects have worked on understanding; however, their focus of research was to define the structure of information (lexical meaning, world representation, etc.) necessary for the presumed "understanding" (or paraphrasing) of certain sentences of the texts. Still, these projects were not oriented for content analysis of for information retrieval and mainly were limited to a specific domain. These AI. systems aimed at exploring complex structured information; this task does not necessarily demand a full understanding of the text. We should stress that many users in the field of the humanities and social research prefer the computer not to "understand" the text. What they are looking for is much more an intermediary structured information that would permit more exhaustive or complex analysis, such as thematic, lexical, textual structure, etc.

Though language itself, is a system that consists of complex systems of communication, human minds can handle with the problems, which is reflected not only in structures of the languages, but also in semantics of natural language. We have to accept that, there are many languages and dialects, and all languages consist of a linguistic system- unique set of grammar and syntax rules, words, idioms, collocations, phraseological units, terms and slangs. Besides, in communication process people can rely on their psychological processes as perceptions, judgments, values and intelligence. 
Unluckily, human level capabilities of understanding are not processed in machine level yet, as they do not have human way of thinking and analyze language for its meaning.

But it is necessary to speak of brains as though they manufacture thoughts the way that humans do. The difference is that brains use processes that change themselves-and this means we cannot separate such processes from the products they produce. In particular, brains make memories, which change the ways we will subsequently think.

A lot of work has been done to get computers closer to a human-level understanding of language. Nowadays we see some advances in language understanding systems, as they are moving closer to remarkable capabilities concerning natural language. NLP during decades, helps to understand, interpret human language. Some authors deeply believe that "NLP can be viewed as the bridge between machine language and the natural language of human speech, enabling machines to interpret and translate their language to human language by strictly following internal communication protocols.

Undoubtedly Natural Language Processing have removed many of the communication barriers between humans and computers by translating machine language into human language, and by creating opportunities for humans to accomplish tasks that were impossible before.

Chomskyan linguistic ideas about formal grammar provided philosophers with a new framework for exploring human language and mind. It was developed at the same time with the algorithms for parsing programming languages in 1960-s and then turned out to be only somewhat indirectly useful for NLP. Vilém Novák proves that much has been done in understanding the structure of a language, especially in the phonetic and syntactic aspects. Less, however, is done for understanding of semantics.

Last few years significant advances have occurred in processing semantics- the content (or meaning) of sentences, as for conveying relevant and sufficient information. Language understanding systems are moving closer to remarkable capabilities as it regards to natural language.Less, however, the interpretation capabilities of a language-understanding system depending on the semantic theory is not fully accepted and satisfactory in all respects. Still, competing semantic theories of language have specific trade-offs in their suitability on the basis of computer-automated semantic interpretation and they are often based on set theories, but not on a semantical logic, which is a main bias in translation process. The understanding of a natural language text requires that a reader-human or computer program be able to resolve ambiguities at the syntactic and lexical levels; it also requires that a reader be able to recover that part of the meaning of a text which is over and above the collection of meanings of its individual sentences taken in isolation. The experimental program reported in Cercone, explores the nature and computational use of meaning representations for word concepts in the context of a natural language understanding system. Word meanings are represented as extended semantic networks and are accessed via a tiny (approximately 200 words) lexicon.

The research we report on in this paper is oriented towards the understanding of some aspects of the semantics involved in the processing of natural language. Mainly the content, logical status of the meaning representation, the compositional processes and the inference rules underlying the sentences in translation processes.

In this paper we process some phenomena of the semantics of natural language using fuzzy sets toward the understanding of certain semantic aspects in computer text processing (words and meaning representation and inference patterns). The request here is much more related to an exploration of understanding than to a translation, paraphrase, deduction, etc.

Some systems have attempted to explore such higher levels of structured information through the paradigms of artificial intelligence. But often they failed, because the paradigm was not applicable on understanding of semantics. More even, how could we provide the real understanding for which we have but a small idea of their content.

We have tried to find out the way out and cope with the vagueness in the models of natural language semantics, meaning of separate lexical units as well as the sentences. Fussy set theory is a mathematical theory, which can provide us with methods and tools for coping with some understanding of certain semantic aspects in computer text processing (words and meaning representation and inference patterns).

Problems, constraints, wishes and challenges for semantic issues

In below mentioned sentences by systematic function of the meaning of the parts can be easily comprehended by human brains. Unfortunately, computers cannot grasp the relationship between the meaning of a sentence and the meanings of its components and fail in understanding of semantics. For example, given a natural language phrase, word combination changes its relevant meaning, how can 
we guess it in such a manner as to find in it the pertinent meaning in the specific context? (E.g. if a sentence has a word combination. The sentence constitutes a semantic space built of an ordered set of sentences which are of different logical types and which present a specific pattern of coherence expressible in a syntactic manner.

You have to cut down on chocolate. It means you have to reduce chocolate.

He understood that he blew it. He understood that he lost the chance.

Wait here till his anger blows over. Wait here till his anger passes.

The bottom line is; I don't have enough money. The main result is; I don't have enough money.

A new approach to semantic interpretation in natural language understanding is described, together with mechanisms for both lexical and structural disambiguation that work in concert with the semantic interpreter. An important concept that should be considered;

- syntax vs semantics

- Word order

- Syntactically well-formed but it is not clear what meaning is

- Semantically contradictory

- Syntactically correct, semantically different

- Formal semantics

- nature of language

Wood in his dissertation mentioned that, for procedural semantics of a natural language, input sentences are translated into procedure call that retrieves information from a database, and the meaning of a sentence was identified with the corresponding procedure call.

Fussy set theory is a mathematical theory, which can provide us with methods and tools for coping with some understanding of certain semantic aspects in computer text processing (words and meaning representation), but not always successful to cope with the vagueness in the models of natural language semantics, meaning of separate lexical units as well as the sentences.

In the theme, the connection to linguistics and the approaches adopted within NLP, the tension predominant view in theoretical linguistics. Nowadays, there is a strong need to understand the (human) contextual capability and uncertainty in the language by a system, as the content analysis of for information retrieval and mainly were limited to a specific domain. There are different ambiguities in understanding.

The symbolic, linguistically based systems sometimes are proved to be of little use when it processes less restricted text.

I bought my wife a new car. She called and said, 'There's water in the carburettor'. I said, 'Where's the car?' She said, 'In the lake.' (Henny Youngman)

A good wife always forgives her husband when she's wrong. (Milton Berle)

I was married by a judge. I should have asked for a jury. (George Burns)

NLP has turned out to be impossibly hard to do for more than very limited domains: the term AI-complete is sometimes used (by analogy to NP-complete), meaning that we'd have to solve the entire problem of representing the world and acquiring world knowledge.

The processing system must exploit the distinctive linguistic properties of the appropriate sublanguage; in fact, a precise description of these properties, incorporated into a sublanguage grammar and lexicon, is what enables the system to build a representation of the information (meaning) conveyed by the text. Sublanguages which appear insufficiently closed for semantic processing often do not carry an important component of information which is encoded in correct linguistically well-behaved way.

When semantic vagueness of meaning in natural language may be an urgent obstacle in machinery natural language, fuzzy logic can be able to handle with uncertainty, incomplete information and limitation inherent in natural language.

For Zadeh. «Computing with Words opens the door to a to a wide ranging enlargement of the role of natural languages in science and engineering». Today, computing with words must still be done using numbers, and, therefore, numeric intervals must be associated with words. An earlier paper [Mendel (1999)] reported on an empirical study that was performed to determine how the scale 0-10 can be covered with words (or phrases). One of the most striking conclusions drawn from this processed data is: linguistic uncertainty appears to be useful in that it lets us cover the $0-10$ range with a much smaller number of terms than without it.

Essentially, CW is a system of computation in where the objects of computation are words, phrases and propositions and they are drawn from a natural language. The propositions are the main 
conveyers of information. It must be remarked that $\mathrm{CW}$ is the only system of computation which offers a capability to compute with information described in a natural language. The Probability distribution is described in words through fuzzy if-then rules.

\section{REFERENCES}

1. Aliev R.A., Fazlollahi B., Aliyev R.R..: Soft Computing and its Applications in Business and Economics, Springer-Verlag Berlin Heidelberg (2004).

2. Colmerauer A.: Metamorphosis Grammars, in: L. Bolt (ed.), Natural Language Communication with Computers, Springer, New York, pp. 133-187, (1978).

3. Copestake A..: Natural Language Processing, 8 Lectures (aac@cl.cam.ac.uk) http://www.cl.cam.ac.uk/users/aac/2004

4. Desikan, B. S..: Natural Language Processing and Computational Linguistics. (2018).

5. Natural language processing.: From Wikipedia, the free encyclopedia.

6. Novák V.: An Introduction to Fuzzy Logic Applications in Intelligent Systems Fuzzy Sets in Natural Language Processing, pp 185-200, (1989).

7. Paul P. Wang.: Computing with words, New York, John Wiley \& Sons. Inc. pp 35-67, (2001).

8. Tsujii, J.: Computational Linguistics and Natural Language Processing. Lecture Notes in Computer Science, pp.52-67, (2011).

9. Yager, R. R..: On the retranslation Process in Zadeh's Paradigm of the Computing with Words. IEEE Transactions on systems, man, and Cybernetics- Part B: Cybernetics, 34(2), (2004).

10. Zadeh L.A.: Toward a perception-based theory of probabilistic reasoning with imprecise probabilities J. Statist. Planning Inference. vol.10, pp. 233-264, (2002).

11. Zadeh L.A.: From computing with numbers to Computing with words- from Manipulation of Measurements to Manipulation of Perception. Wang P (eds) Computing with word. John Wiley \& Sons. Inc. pp 35-67, (2001).

12. Zadeh L.A.: Test-score semantics for natural languages and meaning representation via PRUF, Emprical Semantics. Rieger B (eds) Germany: Brockmyer, pp 198-211, (1981).

13. Zadeh L.A.: What Computing with Words Means to Me. Article in IEEE Computational Intelligence Magazine (2010).

14. Mendel, J. M., "An Architecture for Making Judgments Using Computing With Words," Int. J. Appl. Math. Comput. Sci., vol. 12, No. 3, pp. 325-335, 2002. 